\title{
机械设计制造及其自动化中计算机技术的应用
}

张 龙

郑州飞机装备有限责任公司，河南 郑州 450000

\begin{abstract}
[摘要]在最近的几年时间里，在我国综合国力的不断增强的影响下，使得各个领域都得到了良好的发展。机械设计制造领域 最为突出的特点就是复杂性和繁琐性, 就以往陈旧模式的机械制造情况来说, 工作持续时间长, 质量差, 工作量大的特点十 分明显，但是将计算机技术引用到机械制造之中能够有效的提升机械制造的效率和准确性，从而能够为社会的稳定和谐发展 创造良好的基础。这篇文章主要绕围绕机械设计制造以及其自动化中计算机技术的切实运用展开深入的分析研究，并针对性 的对其未来发展趋势进行了探究，希望能够对机械设计制造行业的稳定发展有所帮助。
\end{abstract}

[关键词]机械设计制作及其自动化; 计算机技术; 应用

DOI：10.33142/aem.v2i2.1612 中图分类号: TH122;TP29-A5;TH16 文献标识码: A

\section{Application of Computer Technology in Mechanical Design and Manufacture and its Automation}

\author{
ZHANG Long \\ Zhengzhou Aircraft Equipment Co., Ltd., Zhengzhou, Henan, 450000, China
}

\begin{abstract}
In recent years, under the influence of the continuous enhancement of China's comprehensive national strength, all fields have been well developed. The most prominent feature in the field of mechanical design and manufacturing is complexity and triviality. As far as the past old mode of mechanical manufacturing is concerned, the features of long duration, poor quality and heavy workload are obvious. But the application of computer technology to mechanical manufacturing can effectively improve the efficiency and accuracy of mechanical manufacturing, so as to create a good foundation for the stable and harmonious development of society. This article mainly around the mechanical design and manufacturing and the practical application of computer technology in its automation to carry out in-depth analysis and research, and targeted to explore its future development trend, hoping to be helpful for the stable development of the mechanical design and manufacturing industry.
\end{abstract}

Keywords: mechanical design, manufacture and automation; computer technology; application

\section{引言}

就当前社会发展形势来看, 我国在机械制造行业的投入在不断的增加, 从而使得机械制造行业的整体水平逐渐的 提升, 我国工厂机械设计制造逐渐的从以往的人工操作朝着自动化的方向迈进。机械制造行业的自动化发展, 使得计 算机在机械产品自动化设计制造中的作用越发的凸现出来。但是因为现如今我国机械设计制造以及自动化中所引用的 计算机技术并没有达到成熟的水平，就目前的状况来看与其他发达国家的专业水平还存在明显的差距，正是因为这一 问题的存在, 对我国机械设计制造的快速发展造成了一定的限制。所以, 我们务必要加大力度针对机械设计以及其自 动化中计算机技术的深入研究和创新, 从根本上提升我国机械设计和自动化制造中的计算机技术的水平, 为我国机械 制造行业的自动化发展提供有利的支持。

\section{1 机械设计制造及自动化实质}

经过对大量的专业文献进行综合研究分析我们总结出, 机械设计制造以及自动化技术具有较强的综合性, 其不但 能够引用到不同的机械和设备的设计制造工序之中, 并且在针对生产能够实现管控工作。现下, 科学技术快速发展, 使得大量科技成果被研发出来, 并被人们切实的引用到了各个领域之中, 取得了非常可喜的成效, 尤其是机械设备制 造以及自动化生产行业发展十分的迅速, 并且在我国经济发展中发挥出了重要的影响作用。其次, 计算机与信息技术 被引用到机械设计制造以及自动化中有效的带动了机械设计制造的智能化水平的不断提升，从而为我国机械制造行业 的快速发展打下了坚实的基础。在新的历史阶段中，我国正在向着工业 2.0 方向发展，机械设计制造以及自动化因为 在工业领域具有较强的影响力, 所以受到了人们的广泛关注。在这一形势下, 我们务必要充分结合实际情况以及社会 
发展趋势, 切实的选择恰当的技术来促进机械设计和自动化整体效率和水平, 为我国工业生产和国民经济的快速发展 给予充足的支持。

\section{2 机械设计制造及其自动化中计算机技术实际运用情况}

在当前新的历史是其中，互联网计算机可以说已经成为了人们生活和工作的不可或缺的一个部分。机械设计制造以 及自动化的发展都是离不开科技的进步和社会发展的助力的, 尽管机械设计制造以及自动化中计算机技术整体水平有了 显著的提升, 但是在那些高新技术行业中, 我国的整体实力还显得较为落后。首先, 尽管人们逐渐的认识到了机械设计 制造以及自动化中运用计算机技术所具有的关键影响, 但是还是有大量的企业对计算机的重要性缺少正确的认识, 所以 导致电子计算机不能得到大范围的运用, 企业的生产往往只能够以来人工操作来完成, 这样对于整个行业的发展都是十 分不利的。其次, 当下我国的理念是将机械设计制造以及自动化计算机技术划分为两个分支模块来进行独立的人才培养, 并且暂时没有将二者加以结合, 从而使得既掌握计算机技术也懂得机械设计制造自动化技术的人才少之又少, 无法满足 行业发展的实际需要。社会的快速发展, 需要我们打破传统的理念的束缚, 要对落后的教育模式进行优化和创新, 这样 才能彻底的扭转机械设计制造以及自动化计算机运用的落后局面, 从而推动整个行业的良好发展。

\section{3 机械设计制造及其自动化中计算机技术的具体应用}

\section{1 计算机辅助技术地应用}

机械设计制造及自动化最为突出的特点就是具有较强的综合性, 正是因为这一特点从而导致机械制造工序十分的 复杂, 并且往往会遇到各种突发问题。在科学技术不断发展的带动下, 机械设计制造及自动化行业内计算机设备的运 用在不断的扩展, 并在实践运用中发挥出了积极的影响作用, 促进了生产制造的效率和质量的不断提升。换句话说, 计算机辅助技术是电子设备中最为基础的功能之一, 将其运用到机械制造以及自动化之中, 能够有效的发挥出增强机 械产品综合性能的作用, 从而可以为生产企业运营生产效率的提升提供一定的辅助, 促使企业获得更加丰厚的经济收 益, 不断的增强自身的综合实力, 这样才能在严峻的市场竞争中长期处在不败的境地。经过综合统计分析我们发现, 计算机辅助技术的作用主要集中在下面几个层面: 首先, 将计算机辅助技术引用到机械设计制造及自动化之中, 能够 为工作人员提供全面的基本信息, 诸如: 机械运行原理、构造以及规格等等, 之后专业人员针对各项信息进行综合分 析, 这样能够准确高效的找到机械优化的方法, 并且能够将机械整体结构星系的呈现出来。其次, 计算机辅助技术的 全面运用, 能够促进机械设计制造以及机械设备自动化水平, 从而能够将原本图纸中抽象的设计形象的展示出来, 这 样更有利于工作人员对产品设计和各项参数加以了解, 并且可以对设备运转中的问题及时的发现, 并进行适当的调整, 保证生产工作的顺利进行。最后, 计算机辅助技术中的重点软件可以借助设计功能表现的优越性, 促使机械设计制造 及自动化产品设计实现自主编辑和完善, 从而可以规避产品多次重复操作的问题发生, 提升生产的效率和质量。

\section{3. $23 \mathrm{D}$ 技术}

将 $3 \mathrm{D}$ 技术切实的引用到机械设计制造及自动化之中, 不但可以有效的提高机械产品的设计效果, 并且能够促进产 品生产的高效性, 对于保证生产效率的提升也具有积极的影响作用。在进行机械化产品设计工作的时候, 运用 3D 技术 可以结合客户需求, 产品规格来创建产品的模型, 这样能够促使设计工作人员全面的对产品设计效果进行观察和分析, 从而精准的判断出设计中存在的问题, 这样才能及时的进行修改, 促使机械产品设计保证达到既定效果。在完成产品 生产工作之后, 利用 $3 \mathrm{D}$ 技术能够实现对产品的各项性能和质量进行综合分析, 从而从根本上对产品质量和实用性加以 保证。就以往机械设计制造模式来说, 往往都是利用物理或者是化学实验的形式来对产品质量进行检测, 而将 $3 \mathrm{D}$ 技术 引用到机械设计制造和自动化之中, 不但可以提升产品质量的检测效率, 还能够缩减检测的成本, 这样就充分的说明 了, 3D 技术在机械设计制造和自动化中的运用效果非常的显著。

\section{3 计算机仿真技术应用}

计算机仿真技术在机械设计及其自动化中的运用非常的普遍, 利用这项技术可以协助所要设计的机械设备创建高 效的三维模型, 设计人员可以结合三维模型各项信息数据对来完成机械设计的优化工作。利用计算机仿真技术创建良 好的三维模型可以协助工作人员来对所有信息数据进行综合分析, 从而能够为整个设计制造行业的良好稳定发展创造 基础。诸如: 在汽车制造行业中, 机械配件设计工作具有较大的难度, 如果没有计算机方针技术的辅助, 那么势必会 增加后期的检测工作量, 并且需要大量的资源的使用, 不但会增加生产成本, 并且所获得的检测结果往往准确性较差。 而在将计算机仿真技术加以切实的运用, 创建仿真模型可以结合实际情况对机械生产状态加以模拟, 这样有利于工作 
人员高效准确的判断出机械设计中存在的问题，提升机械设计的效果。

\section{4 机械设计制造及自动化计算机技术应用未来前景}

\section{1 智能化的发展趋势}

在人工智能快速发展的推动下, 促进了机械设计制造机自动化的智能化发展, 智能化已经充斥到了人们生活和工 作的各个角落。诸如: 智能电子设备, 智能机器人, 智能家用电器等等。在这种社会发展的形势下, 势必会推动计算 机技术在机械设计制造及自动化中的切实运用向着智能化的方向过渡, 否则必然会在社会快速发展的浪潮中被淹没。 当下, 我国在智能化研发方面投入了大量的人力物力, 这样也为机械行业和计算机技术的发展带来了机遇。利用计算 机信息来对人工智能加以模拟，从而会对机械设计制造智能化发展起到良好的促进作用。 ${ }^{[5]}$

\section{2 微型化}

尽管我国科学技术水平发展十分的迅速, 但是与其他发达国家相比较来看, 还存在较为明显的滞后性, 所以导致 我国当前机械产品整体规格较大, 并在实际使用性能方面也存在诸多的问题, 很显然以及不能再满足当下社会发展的 实际需要了, 所以为了更好的为社会发展创造良好的基础, 机械产品设计工作务必要重视规格的微型化转变。并且要 在上述工作的基础上, 针对产品整体结构以及性能软件加以综合优化, 为我国社会的稳定发展创造良好的条件。

\section{3 网络化}

网络技术的快速发展, 推动了监控技术的进步, 这样就为机械产品的传播范围的不断延伸创造了良好的条件。在 互联网平台上, 信息的传输更加的高效, 机械制造企业可以利用互联网平台对生产的机械产品进行宣传, 不但可以提 升企业的销售, 并且可以提高企业知名度。

\section{5 结语}

总的来说, 将最前沿的计算机技术切实的引用到机械设计制造及其自动化之中, 不但可以带动整体工作效率和质 量的提升, 并且能够增强其综合能力。在计算机技术快速发展的带动下, 促使我国机械制造行业正在向着智能化、经 济化的方向转变发展。

\section{[参考文献]}

[1]沈平牶. 研究机械设计制造及其自动化中的计算机技术运用 [J].建材与装饰, 2018(11):240-241.

[2]郑璐晔,潜俊儒. 机械设计制造及其自动化中计算机技术的应用 $[\mathrm{J}]$. 现代制造技术与装备, 2017 (11) : 159-160.

[3] 李珑. 计算机技术在机械设计制造及其自动化中的应用分析 [J]. 科学技术创新, 2017 (30): 125-126.

[4]高林. 机械设计制造及其自动化中计算机技术的应用分析 [J].居舍, 2017 (28): 124 .

[5]柯政.机械设计制造及其自动化中计算机技术的应用 $[\mathrm{J}]$. 科技风,2017 (18) : 168 .

[6]刘建军. 机械设计制造及其自动化中计算机技术的应用 [J]. 时代农机, 2017, 44(07): 22-26.

作者简介: 张龙 (1987-), 男, 毕业于西北农林科技大学, 机械设计及理论专业, 就职于郑州飞机装备有限责任公司, 设计员, 中级职称。 